\title{
Excessive Sucking
}

National Cancer Institute

\section{Source}

National Cancer Institute. Excessive Sucking. NCI Thesaurus. Code C87073.

A symptom of gastrointestinal dysfunction seen in neonates who have been exposed to drugs during pregnancy; often accompanied by excessive sucking of fists, fingers and thumbs. 